\title{
Trinômes irréductibles résolubles sur un corps de nombres
}

\author{
par \\ Julien Angeli (Limoges)
}

1. Introduction. Le problème de la détermination des trinômes $X^{n}-a X^{m}+b$ à coefficients dans un corps de nombres $k$, avec $n$ et $m$ premiers entre eux, et dont le groupe de Galois est un sous-groupe $G$ de $S_{n}$ donné, se ramène à la recherche de points $k$-rationnels sur une certaine courbe algébrique, dépendant de $G, n$ et $m$. Le calcul du genre de cette courbe présente alors un intérêt important : on sait que l'on a trois comportements très différents lorsque $g=0, g=1$ ou $g \geq 2$. Dans ce dernier cas, la conjecture de Mordell, démontrée par Faltings [6], affirme qu'il n'existe qu'un nombre fini de points rationnels sur la courbe. À homothétie des racines près, il n'existera alors qu'un nombre fini de tels trinômes.

Pour tout entier $n$ premier, on se propose de déterminer le genre de la courbe correspondant au groupe $G=\operatorname{AGL}(1, n)$ des transformations affines sur le corps à $n$ éléments, vu comme un sous-groupe de $S_{n}$. On recherche de cette façon les trinômes irréductibles et résolubles : c'est une propriété bien connue que tout polynôme irréductible et résoluble de degré $n$ a son groupe de Galois inclus dans le groupe affine AGL(1,n) (cf. [1]). Le cas $n=5$ est déjà connu dans la littérature [9]. Pour $m=1$, le genre de la courbe vaut 0 et on a une famille paramétrée de trinômes résolubles : les $\left(4 u^{2}+16\right) x^{5}+\left(5 u^{2}-5\right) x+\left(4 u^{2}+10 u+6\right)($ cf. [11]). Pour $m=2$, le genre vaut 1 . La courbe est une courbe elliptique de rang 0 dont les points $\mathbb{Q}$ rationnels donnent exactement 5 trinômes de $\mathbb{Q}[t]$ résolubles (à équivalence près) : $5 x^{5}-10 x^{2}-1, x^{5}-100 x^{2}-1000, x^{5}-5 x^{2}-3, x^{5}-5 x^{2}+15$, $x^{5}-25 x^{2}-300$. On montre ici que pour tout $n>5$ premier, le genre cherché est toujours supérieur à 2 et qu'il n'existe donc qu'un nombre fini, à équivalence près, de trinômes irréductibles résolubles de degré $n$ sur tout corps de nombres $k$.

Quelques exemples similaires de calculs du genre sont déjà connus. L'article de A. Schinzel [8] traite le cas où $G$ est non transitif. Une expression

2000 Mathematics Subject Classification: 11R32, 11S15, 12F12. 
générale du genre est donnée lorsque $G=S_{p} \times S_{n-p}$. Il est ensuite possible de déterminer quels sont les triplets $(n, m, p)$ pour lesquels le genre est nul, d'en déduire quels sont les trinômes $X^{n}-a X^{m}+b$, avec $b^{n-m} a^{-n} \notin \mathbb{Q}$ à coefficients dans $\mathbb{Q}(t)$, qui se factorisent en deux facteurs de degré $p$ et $n-p$, et enfin de donner leur factorisation explicite. En dehors des cas $p=1$, $p=2$ et des triplets $(2 p, p, p)$, on obtient une liste finie de 12 factorisations possibles.

Dans l'article de N. Bruin et N. D. Elkies [2], on donne le genre, égal à 2, puis l'équation, de la courbe correspondant au groupe $G=\operatorname{PSL}(3,2)$, pour $n=7$ et $m=1$. La courbe n'a qu'un nombre fini de points $\mathbb{Q}$-rationnels, et une recherche informatique permet de les déterminer tous. On retrouve ainsi les trinômes déjà connus de Trinks-Matzat $\left(X^{7}-7 X+3\right.$, cf. [10]) et de Erbach-Fischer-McKay $\left(X^{7}-154 X+99\right.$, cf. [5]), auxquels viennent s'ajouter deux nouveaux trinômes. Un travail similaire est effectué pour $n=8, m=1$, et $G=\operatorname{AGL}(3,2)$, le groupe des transformations affines de $\left(\mathbb{F}_{2}\right)^{3}$. La courbe correspondante est elle aussi de genre 2 , et la recherche (non exhaustive) de points rationnels donne quatre trinômes exceptionnels jusque là inconnus.

Un panorama très complet sur les trinômes peut être trouvé sur le site de N. D. Elkies [4].

\section{La courbe $C(\bar{k})$}

2.1. Notations. Dans la suite, $k$ sera un corps de nombres, et $\bar{k}$ sa clôture algébrique. On se donne deux entiers premiers entre eux $n$ et $m$, avec $0<$ $m<n$, et $G$ un sous-groupe quelconque de $S_{n}$. On définit l'ensemble des trinômes $(n, m)$ sur $\bar{k}$ :

$$
\mathcal{T}_{n, m}(\bar{k})=\left\{X^{n}-a X^{m}+b: a, b \in \bar{k}^{\times}\right\} .
$$

On rappelle que le groupe $\bar{k}^{\times}$agit sur $\bar{k}[X]$ et $\mathcal{T}_{n, m}(\bar{k})$ par $\mu . P(X)=$ $\mu^{-n} P(\mu X)$. Cette action laisse le groupe de Galois invariant. Si on se fixe deux entiers $r$ et $s$ tels que $s(n-m)-r n=1$, alors chaque trinôme $X^{n}-$ $a X^{m}+b$ est équivalent à un unique trinôme réduit $X^{n}-t^{r} X^{m}+t^{s}$. On appelle paramètre du trinôme la quantité $t$, qui est égale à $b^{n-m} a^{-n}$.

On définit le trinôme générique $T(X)=X^{n}-t^{r} X^{m}+t^{s} \in \bar{k}(t)[X]$. On note $N$ (respectivement $N_{0}$ ) son corps des racines sur $\bar{k}(t)$ (respectivement $k(t))$. Le groupe de Galois des extensions $N / \bar{k}(t)$ et $N_{0} / k(t)$ est $S_{n}$ (cf. [8]). Comme $G$ est un sous-groupe de $S_{n}$, il agit naturellement sur $N$ (respectivement $N_{0}$ ), et on peut introduire le corps $N^{G}$ (respectivement $N_{0}^{G}$ ) fixé par $G$.

2.2. La courbe $C(\bar{k})$. Les corps $N$ et $N^{G}$ sont des extensions de $\bar{k}$ de degré de transcendance 1 . Or, la catégorie des extensions de degré de transcendance 1 sur $\bar{k}$, dont les flèches sont les $\bar{k}$-morphismes, est équivalente à la 
catégorie des courbes quasi-projectives, dont les flèches sont les morphismes rationnels dominants [7]. Cela nous permet d'introduire les courbes $C(\bar{k})$, $C_{G}(\bar{k})$ et $\mathbb{P}^{1}(\bar{k})$, dont les corps de fonctions respectifs sont $N, N^{G}$ et $\bar{k}(t)$ :

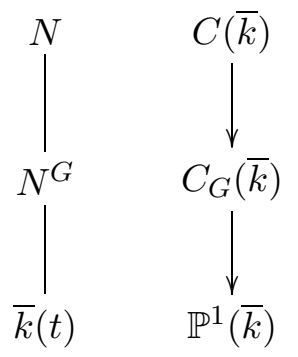

De $C(\bar{k})$, on peut donner un modèle explicite : $\grave{A}$ tout point $\left(\lambda_{0}: \ldots\right.$ $\left.: \lambda_{n-1}\right)$ de $\mathbb{P}^{n-1}(\bar{k})$, on associe la classe d'équivalence du polynôme

$$
\prod_{i=0}^{n-1}\left(X-\lambda_{i}\right)=X^{n}-\sigma_{1} X^{n-1}+\cdots+(-1)^{n-1} \sigma_{n-1} X+(-1)^{n} \sigma_{n}
$$

où $\sigma_{i}$ est la fonction symétrique élémentaire de degré $i$ des coordonnées $\lambda_{0}, \ldots, \lambda_{n-1}$.

On prend pour $C(\bar{k})$ l'ensemble des points de $\mathbb{P}^{n-1}(\bar{k})$ associés aux trinômes de $\mathcal{T}_{n, m}(\bar{k})$, c'est-à-dire la variété algébrique définie par les équations homogènes $\sigma_{i}=0$, pour $1 \leq i<n-m, n-m<i<n$.

Pour se représenter $C_{G}(\bar{k})$, rappelons que $C(\bar{k}), C(k), C_{G}(\bar{k})$ et $C_{G}(k)$ s'identifient respectivement à l'ensemble des places de degré 1 de $N, N_{0}, N^{G}$ et $N_{0}^{G}$. Il y a alors une bijection naturelle entre $C_{G}(\bar{k})$ et l'ensemble des $G$ orbites des points de $C(\bar{k})$ (cf. [3], notamment le théorème 2 du chapitre IV).

2.3. Inertie de l'extension $N / \bar{k}(t)$. On aura besoin de connaître l'inertie de $N / \bar{k}(t)$. C'est un résultat déjà connu $([8])$, que l'on se contente de rappeller :

Proposition 1. Soit $t_{0}=m^{m}(n-m)^{n-m} n^{-n}$. Un trinôme de $\mathcal{T}_{n, m}(\bar{k})$ est inséparable si, et seulement si, son paramètre est égal à $t_{0}$.

On note respectivement $v_{0}, v_{t_{0}}$ et $v_{\infty}$ les places $(t)$-adique, $\left(t-t_{0}\right)$-adique et $(1 / t)$-adique de $\bar{k}(t)$.

Alors $v_{0}, v_{t_{0}}$ et $v_{\infty}$ sont les seules places de $\bar{k}(t)$ qui se ramifient dans $N$.

- Si $w$ est une place de $N$ au-dessus de $v_{0}$, alors le groupe d'inertie $I\left(w \mid v_{0}\right)$ est engendré par le produit disjoint d'un m-cycle et d'un $(n-m)$-cycle.

- Si w est une place de $N$ au-dessus de $v_{t_{0}}$, alors $I\left(w \mid v_{t_{0}}\right)$ est engendré par une transposition.

- Si w est une place de $N$ au-dessus de $v_{\infty}$, alors $I\left(w \mid v_{\infty}\right)$ est engendré par un n-cycle. 
2.4. Paramétrisation des trinômes de groupe de Galois inclus dans $G$. Il existe sur $C(\bar{k})$ une fonction rationnelle $\tau: C(\bar{k}) \rightarrow \mathbb{P}^{1}(\bar{k})$ qui à tout point de la courbe associe le paramètre du trinôme correspondant; sa valeur est $(-1)^{n} \sigma_{n}^{n-m} \sigma_{n-m}^{-n}$.

La fonction $\tau$ est invariante sous l'action de $S_{n}$, on dispose donc sur $C_{G}(\bar{k})$ ou $\mathbb{P}^{1}(\bar{k})$ de la fonction image de $\tau$, qu'on notera encore $\tau$.

Il est clair que l'image par $\tau$ d'un point $k$-rationnel $\left(\lambda_{0}: \ldots: \lambda_{n-1}\right)$ de $C(\bar{k})$ est le paramètre d'un trinôme $\prod_{i=0}^{n-1}\left(X-\lambda_{i}\right)$ scindé sur $k$, c'est-à-dire de groupe de Galois trivial. Plus généralement, on a la propriété suivante :

Proposition 2. Les paramètres des $k$-trinômes séparables, dont le groupe de Galois est inclus dans $G$, sont exactement les $\tau(P)$ différents de $0, t_{0}$ et $\infty$, pour $P \in C_{G}(k)$.

On utilise le lemme suivant :

Lemme 1. Soit $P \in k(t)[X]$ et $\theta \in k$. Soit $N$ le corps des racines de $P$ sur $k(t), v$ une place de $N$ au-dessus de la place $(t-\theta)$-adique de $k(t)$, et $\widehat{N}$ le complété de $N$ pour la place $v$. On suppose que la réduction $P^{\mathrm{res}}$ de $P$ modulo $v$ est un polynôme séparable de $k(t)$. Alors le corps résiduel $\widehat{N}^{\mathrm{res}}$ est le corps des racines de $P^{\text {res }}$ sur $k$.

Preuve. Le polynôme $P$ est lui aussi séparable, on note $L_{0}, \ldots, L_{n-1}$ ses racines distinctes dans $N$. Il est clair que leurs réductions $\lambda_{0}, \ldots, \lambda_{n-1}$ dans $\widehat{N}^{\text {res }}$ sont des racines de $P^{\text {res }}$. D'après le lemme de Hensel, on peut relever chaque $\lambda_{i}$ en une unique racine de $P$, donc les $\lambda_{i}$ sont distincts, et $\widehat{N}^{\text {res }}$ contient toutes les racines de $P^{\text {res }}$.

Il suffit alors de montrer que $\widehat{N}^{\text {res }}$ est engendré par les $\lambda_{i}$. On peut introduire un élément primitif $u$ de $\widehat{N}$ tel que $u \in k\left[L_{0}, \ldots, L_{n-1}\right]$. Sa réduction $u^{\text {res }}$ modulo $v$ appartient alors à $k\left[\lambda_{0}, \ldots, \lambda_{n-1}\right]$. Choisissons un élément $a^{\text {res }}$ de $\widehat{N}^{\text {res }}$; il se relève en $a=\sum f_{i}(t-\theta) u^{i} \in \widehat{N}$. Les séries $f_{i}$ sont entières ; sinon, en multipliant par $(t-\theta)^{j}$, où $j$ est le plus grand ordre des pôles des $f_{i}$, et en réduisant modulo $v$, on aboutit à une contradiction. Par conséquent, $a^{\text {res }}=\sum f_{i}(0)\left(u^{\text {res }}\right)^{i} \in k\left[\lambda_{0}, \ldots, \lambda_{n-1}\right]$, ce qui démontre le lemme.

Preuve de la proposition 2. Supposons d'abord que $P$ soit un point de $C_{G}(k)$ tel que la quantité $\theta=\tau(P)$ soit différente de $0, t_{0}$ et $\infty$.

Au point $P$ correspond une place $v_{P}$ de $N_{0}^{G}$; on note $\widehat{N_{0}^{G}}$ le complété de $N_{0}^{G}$ pour $v_{P}$. La place $v_{P}$ est au-dessus d'une place $v_{\theta}$ de $k(t)$, et on complète ce dernier pour obtenir le corps $k((t-\theta))$. Enfin, on choisit une place $v$ de $N_{0}$ au-dessus de $v_{P}$, et on complète $N_{0}$ en le corps $\widehat{N_{0}}$. On obtient ainsi une tour de corps complétés : 

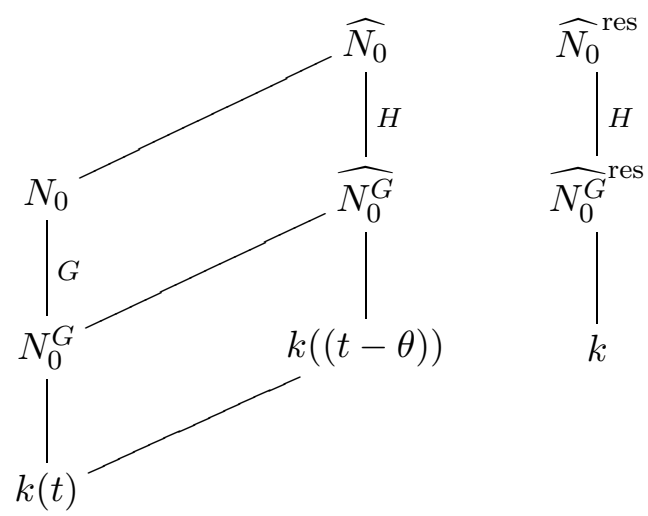

Le groupe de Galois $H$ de l'extension $\widehat{N_{0}} / \widehat{N_{0}^{G}}$ est le sous-groupe de décomposition du groupe de Galois $G$ de $N_{0} / N_{0}^{G}$, pour la place $v$ de $N_{0}$ choisie.

Le groupe de Galois entre les corps résiduels ${\widehat{N_{0}}}^{\text {res }}$ et ${\widehat{N_{0}^{G}}}^{\text {res }}$ est le quotient du groupe de décomposition $H$ par le sous-groupe d'inertie; comme $\theta$ est différent de $0, t_{0}, \infty$, l'extension $\widehat{N}_{0} / \widehat{N}_{0}^{G}$ est non ramifiée, et $\operatorname{Gal}\left(\widehat{N}_{0}{ }^{\text {res }} / \widehat{N}_{0}^{G}{ }^{\text {res }}\right)$ s'identifie à $\operatorname{Gal}\left(\widehat{N_{0}} / \widehat{N_{0}^{G}}\right)=H$.

Le corps résiduel de $\widehat{N_{0}^{G}}$ est égal à $k$ car $P$ est $k$-rationnel, et $\widehat{N}_{0}^{\text {res }}$ est le corps des racines de $X^{n}-\theta^{r} X^{m}+\theta^{s}$ sur $k$.

Ceci démontre un sens de l'équivalence.

Réciproquement, soit $\theta \in k$ tel que le trinôme $X^{n}-\theta^{r} X^{m}+\theta^{s}$ soit séparable, de groupe de Galois sur $k$ inclus dans $G$. On a alors $\theta$ différent de $0, t_{0}$ et $\infty$. Notons $\lambda_{0}, \ldots, \lambda_{n-1}$ les racines de $X^{n}-\theta^{r} X^{m}+\theta^{s}$. Comme précédemment, on introduit les corps complétés. Le point $\left(\lambda_{0}: \ldots: \lambda_{n-1}\right)$ de $C(\bar{k})$ est associé à une place $v$ de $N_{0}$. On appelle $k((t-\theta)), \widehat{N_{0}^{H}}$ et $\widehat{N_{0}}$ les complétés par rapport à $v$ :
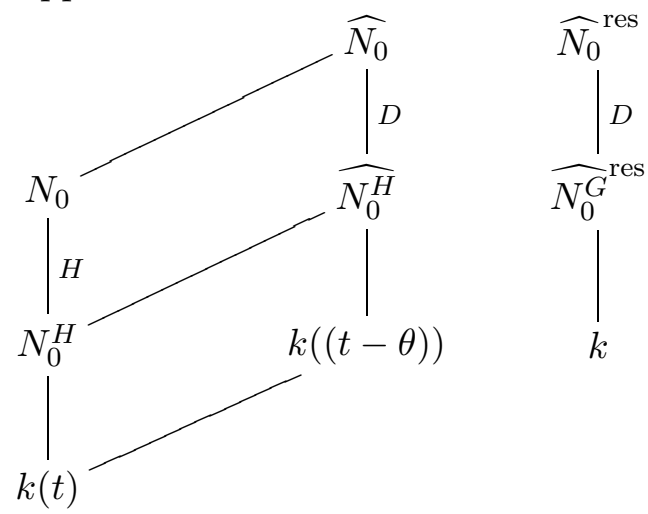

Le corps résiduel ${\widehat{N_{0}}}^{\text {res }}$ est le corps des racines sur $k$ du trinôme $X^{n}-$ $\theta^{r} X^{m}+\theta^{s}$. 
Le groupe de Galois entre $\widehat{N}_{0}^{\text {res }}$ et ${\widehat{N_{0}^{G}}}^{\text {res }}$ est aussi le groupe de décomposition $D=\operatorname{Gal}\left(\widehat{N_{0}} / \widehat{N_{0}^{H}}\right)$; c'est un sous-groupe de $H=\operatorname{Gal}\left(\widehat{N}_{0}^{\text {res }} / k\right)$. On en déduit que ${\widehat{N_{0}^{G}}}^{\text {res }}=k$.

Par conséquent, la restriction de la place $v$ est de degré 1 sur $N_{0}^{H}$, elle est donc a fortiori de degré 1 sur le corps $N_{0}^{G}$, qui est inclus dans $N_{0}^{H}$. Par conséquent, le point $P$ de $C_{G}(\bar{k})$, image de $\left(\lambda_{0}, \ldots, \lambda_{n-1}\right)$, est $k$-rationnel. Comme $\theta=\tau\left(\left(\lambda_{0}: \ldots: \lambda_{n-1}\right)\right)=\tau(P)$, cela conclut la démonstration.

3. Calcul et minoration du genre. Désormais $n$ est un nombre premier, et $G$ est le groupe affine $\operatorname{AGL}(1, n)$ sur le corps $\mathbb{F}_{n}$. Remarquons qu'il existe plusieurs choix possibles d'un plongement de $G$ dans $S_{n}$, qui correspondent à des numérotations différentes des racines. Dans la suite, on suppose que ce choix a été fait une fois pour toutes.

Dans cette section, on calcule le genre du corps $N^{G}$, et donc de la courbe $C_{G}(\bar{k})$. Pour cela, on calcule les groupes d'inertie de l'extension $N^{G} / \bar{k}(t)$, on obtient les indices de ramifications comme cardinaux de ces groupes, et on utilise la formule de Riemann-Hurwitz.

3.1. Structure de $G$. Le groupe $G$ des transformations affines sur $\mathbb{F}_{n}$ est un groupe d'ordre $n(n-1)$. Il est la réunion des sous-groupes d'intersections triviales suivants :

(i) le groupe cyclique d'ordre $n$ des translations,

(ii) les $n$ groupes cycliques d'ordre $n-1$ des transformations affines fixant un point $a \in \mathbb{F}_{n}$ (c'est-à-dire des homothéties de centre $a$ ).

Dans la suite, $v$ est la place $v_{0}, v_{t_{0}}$ ou $v_{\infty}$ de $\bar{k}(t), w$ est une place de $N^{G}$ au-dessus de $v$, et $u$ une place de $N$ au-dessus de $w$. On a

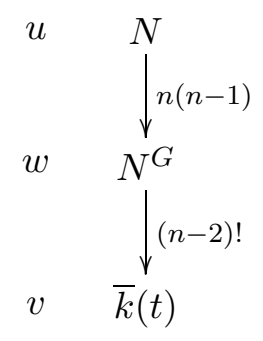

3.2. Nombre de branchement au-dessus de $v_{\infty}$. Il y a $(n-1)$ ! places $u$ de $N$ au-dessus de $v_{\infty}$, et leurs groupes d'inertie $I\left(u \mid v_{\infty}\right)$ sont des groupes cycliques d'ordre $n$. De plus, $I(u \mid w)=I\left(u \mid v_{\infty}\right) \cap G$.

Il y a $(n-2)$ ! groupes cycliques d'ordre $n$ dans $S_{n}$. Chacun d'entre eux est le groupe d'inertie $I$ de $n-1$ places. En effet, si $I$ est le groupe d'inertie de $u_{1}, \ldots, u_{k}$, et si $\sigma \in \operatorname{Gal}(N / L)=G$, alors $\sigma^{-1} I \sigma$ est le groupe d'inertie 
de $u_{1} \circ \sigma, \ldots, u_{k} \circ \sigma$, et l'action du groupe de Galois sur les places au-dessus de $v_{\infty}$ est transitive.

L'un de ces $(n-2)$ ! groupes est inclus dans le groupe affine $G$ (il s'agit du sous-groupe des translations), tous les autres intersectent $G$ trivialement.

Donc $n-1$ places $u$ sont ramifiées au-dessus de $w$, d'indice $e(u \mid w)=n$, et les $(n-1)((n-2) !-1)$ places $u$ restantes sont non ramifiées au-dessus de $w$.

Par conséquent, si on examine la ramification entre $N^{G}$ et $k(t)$, on voit qu'il y a une place $w$ non ramifiée au-dessus de $k(t)$, et $((n-2) !-1) / n$ places $w$ ramifiées, avec un indice $e\left(w \mid v_{\infty}\right)=n$.

On en déduit le nombre de branchement :

$$
B_{\infty}=\frac{1}{2} \sum_{w}(e-1)=\frac{1}{2} \frac{(n-2) !-1}{n}(n-1) .
$$

3.3. Nombre de branchement au-dessus de $v_{t_{0}}$. Cette fois, les groupes d'inertie $I$ sont d'ordre 2. Le groupe $G$ ne contenant pas de transposition, l'intersection $I\left(u \mid v_{t_{0}}\right) \cap G$ est toujours triviale. Donc il n'y a pas de ramification entre $N$ et $N^{G}$, toute la ramification se situe entre les corps $N^{G}$ et $k(t)$. On en déduit qu'il y a $(n-2) ! / 2$ places $w$ ramifiés au-dessus de $v_{t_{0}}$, d'indice $e\left(w \mid v_{t_{0}}\right)=2$, ce qui donne le nombre de branchement

$$
B_{t_{0}}=\frac{(n-2) !}{4} \text {. }
$$

3.4. Nombre de branchement au-dessus de $v_{0}$, quand $m>1$. On distingue deux cas pour le calcul de $B_{0}$. Le cas simple est celui où $m>1$. En effet, les groupes d'inerties sont alors engendrés par le produit disjoint d'un $m$-cycle et d'un $(n-m)$-cycle. Comme la seule transformation affine fixant deux points est l'identité, l'intersection de $G$ et d'un tel groupe d'inertie est toujours triviale.

Comme il y a $n ! / m(n-m)$ places $u$ de $N$ au-dessus de $v_{0}$, et comme toute la ramification se situe entre $N^{G}$ et $k(t)$, il y a $(n-2) ! / m(n-m)$ places $w$ au-dessus de $v_{0}$, d'indice $e\left(w \mid v_{0}\right)=m(n-m)$. On obtient donc

$$
B_{0}=\frac{(n-2) !}{2 m(n-m)}(m(n-m)-1) .
$$

3.5. Nombre de branchement au-dessus de $v_{0}$, quand $m=1$. Cette fois, les groupes d'inertie sont des groupes cycliques d'ordre $n-1$, et leur intersection avec $G$ n'est pas toujours triviale. Pour tout $d$ divisant $n-1$, on veut dénombrer les groupes d'inertie $I$ dont l'intersection avec $G$ est d'ordre $d$. Notons $\alpha_{d}$ leur nombre.

Soit $H$ un sous-groupe cyclique de $G$ engendré par une permutation $\sigma$, produit de $(n-1) / d d$-cycles disjoints.

- Le groupe $H$ contient $\phi(d)$ générateurs. 
- Chacun de ces générateurs a $\prod_{k=1}^{(n-1) / d-1}(n-1-k d)$ racines $(n-1) / d$ ièmes (qui sont des $(n-1)$-cycles).

- Chaque groupe $I$ contient $\phi(n-1)$ générateurs, qui sont ces $(n-1)$ cycles.

Par conséquent, $H$ est inclus dans

$$
\frac{\phi(d)}{\phi(n-1)} \prod_{k=1}^{(n-1) / d-1}(n-1-k d)
$$

groupes $I$, quantité égale après simplification à

$$
\frac{\phi(d)}{\phi(n-1)} d^{(n-1) / d-1}\left(\frac{n-1}{d}-1\right) ! .
$$

Comme il existe $n$ tels groupes $H$ dans $G$, on en déduit l'égalité

$$
\sum_{d|e| n-1} \alpha_{e}=n \frac{\phi(d)}{\phi(n-1)} d^{(n-1) / d-1}\left(\frac{n-1}{d}-1\right) !
$$

On applique la formule d'inversion de Möbius pour obtenir

$$
\alpha_{d}=\sum_{d|e| n-1} n \frac{\phi(e)}{\phi(n-1)} e^{(n-1) / e-1}\left(\frac{n-1}{e}-1\right) ! \mu\left(\frac{e}{d}\right) .
$$

Chaque groupe $I$ est le groupe d'inertie de $\phi(n-1)$ places $u$ de $N$, ramifiées au-dessus de $w$, d'indice $e(u \mid w)=|I \cap G|$.

Il y a donc $\alpha_{d} \phi(n-1)$ places de $N$ d'indice $e=d$ au-dessus de $N^{G}$.

$\grave{A}$ ces places correspondent $\alpha_{d} \phi(n-1) d / n(n-1)$ places $w$ de $N^{G}$, d'indice de ramification $(n-1) / d$ au-dessus de $v_{0}$, et on peut calculer le nombre de branchement correspondant à la place $v_{0}$ :

$$
\begin{aligned}
B_{0} & =\frac{1}{2} \sum_{d \mid n-1} \alpha_{d} \phi(n-1) \frac{d}{n(n-1)}\left(\frac{n-1}{d}-1\right) \\
& =\frac{1}{2} \sum_{d|e| n-1} \phi(e) e^{(n-1) / e-1}\left(\frac{n-1}{e}-1\right) ! \mu\left(\frac{e}{d}\right)\left(1-\frac{d}{n-1}\right) \\
& =\frac{1}{2} \sum_{e \mid n-1} \phi(e) e^{(n-1) / e-1}\left(\frac{n-1}{e}-1\right) ! \sum_{d \mid e} \mu\left(\frac{e}{d}\right)\left(1-\frac{d}{n-1}\right) .
\end{aligned}
$$

En posant $f=e / d$, la somme $\sum_{d \mid e} \mu(e / d)(1-d /(n-1))$ s'écrit

$$
\sum_{f \mid e} \mu(f)-\frac{e}{n-1} \sum_{f \mid e} \frac{\mu(f)}{f} .
$$

Le terme $\sum_{f \mid e} \mu(f)$ vaut 1 si $e=1$, et 0 sinon. Le terme $U(e):=\sum_{f \mid e} \mu(f) / f$ est égal à $\phi(e) / e$. En effet, $U$ est une fonction multiplicative, et $U\left(p^{k}\right)=$ 
$1-1 / p=\phi\left(p^{k}\right) / p^{k}$. On aboutit à l'expression suivante de $B_{0}$ :

$$
B_{0}=\frac{1}{2}(n-2) !-\frac{1}{2(n-1)} \sum_{e \mid n-1} \phi(e)^{2} e^{(n-1) / e-1}\left(\frac{n-1}{e}-1\right) !
$$

3.6. Genre de la courbe et minoration

Proposition 3. Si $m=1$, alors le genre $g$ de la courbe est égal à $g=\frac{1}{2}+\frac{1}{2 n}+(n-2) !\left(\frac{1}{4}-\frac{1}{2 n}\right)-\frac{1}{2(n-1)} \sum_{e \mid n-1} \phi(e)^{2} e^{\frac{n-1}{e}-1}\left(\frac{n-1}{e}-1\right) !$.

Si $m>1$, alors le genre $g$ vaut

$$
g=\frac{1}{2}+\frac{1}{2 n}+(n-2) !\left(\frac{1}{4}-\frac{1}{2 n}-\frac{1}{2 m(n-m)}\right) .
$$

Dans tous les cas, on a $g>1$ pour tout premier $n>5$.

Preuve. L'expression du genre s'obtient à partir de la formule de Riemann-Hurwitz :

$$
g=1-\left[S_{n}: G\right]+B_{\infty}+B_{t_{0}}+B_{0} .
$$

La minoration de $g$ dans le cas $m>1$ s'obtient simplement : On a $2 n \geq 12$, $2 m(n-m) \geq 10$ et $(n-2) ! \geq 24$, donc

$$
g>\frac{1}{2}+24\left(\frac{1}{4}-\frac{1}{12}-\frac{1}{10}\right)>1 .
$$

Dans le cas $m=1$, on majore le terme $\phi(e)^{2} e^{(n-1) / e-1}\left(\frac{n-1}{e}-1\right)$ ! pour $e>1$ :

$$
\phi(e)^{2} e^{(n-1) / e-1}\left(\frac{n-1}{e}-1\right) !<e\left(e^{1 / e}\right)^{n-1}\left(\frac{n-1}{2}-1\right) ! .
$$

Une étude élémentaire de la fonction $x \mapsto x^{1 / x}$ montre que $e^{1 / e} \leq 3^{1 / 3}$. Donc

$$
\phi(e)^{2} e^{(n-1) / e-1}\left(\frac{n-1}{e}-1\right) !<\left(\frac{n-1}{2}-1\right) ! 3^{(n-1) / 3}(n-1) .
$$

Quand $n \geq 13$, ce produit est inférieur (terme à terme) à $2(n-3)$ !. Alors

$$
g \geq \frac{1}{2}+\frac{1}{2 n}+(n-2) !\left(\frac{1}{4}-\frac{1}{2 n}\right)-\frac{1}{2(n-1)}\left((n-2) !+\frac{n-2}{2} \cdot 2(n-3) !\right)
$$

et

$$
g>(n-2) !\left(\frac{1}{4}-\frac{1}{2 n}-\frac{1}{n-1}\right) \geq 2 .
$$

Pour conclure la démonstration, il ne reste qu'à vérifier la valeur de $g$ pour les petites valeurs de $n$ : 


\begin{tabular}{c|c}
$n$ & $g$ \\
\hline 5 & 0 \\
7 & 10 \\
11 & $56058 \quad$ ロ
\end{tabular}

On peut donner finalement le résultat :

ThÉORÈME 1. Pour tout entier premier $n>5$, pour tout corps de nombres $k$, il n'existe qu'un nombre fini, à équivalence près, de $k$-trinômes de degré $n$ irréductibles et résolubles sur $k$.

Preuve. D'après le théorème de Faltings [6], la courbe $C_{G}(\bar{k})$, de genre strictement supérieur à 1 , n'admet qu'un nombre fini de points $k$-rationnels. Donc il n'existe qu'un nombre fini de $k$-trinômes $X^{n}-a X^{m}+b$ de groupe de Galois inclus dans le groupe affine. Comme il n'y a qu'un nombre fini de $m$ possibles, le résultat s'en déduit.

\section{Références}

[1] E. Artin, Galois Theory, Dover, Mineola, NY, 1998.

[2] N. Bruin and N. D. Elkies, Trinomials $a x^{7}+b x+c$ and $a x^{8}+b x+c$ with Galois groups of order 168 and $8 \cdot 168$, in: Lecture Notes in Comput. Sci. 2369, Springer, Berlin, 2002, 172-188.

[3] C. Chevalley, Introduction to the Theory of Algebraic Functions of One Variable, Amer. Math. Soc., New York, 1951.

[4] N. D. Elkies, Trinomials $a x^{n}+b x+c$ with interesting Galois groups, http://www. math.harvard.edu/ ${ }^{\sim}$ elkies/trinomial.html.

[5] D. W. Erbach, J. Fischer and J. McKay, Polynomials with $\operatorname{PSL}(2,7)$ as Galois group, J. Number Theory 11 (1979), 69-75.

[6] G. Faltings, Endlichkeitssätze für abelsche Varietäten über Zahlkörpern, Invent. Math. 73 (1983), 349-366.

[7] R. Hartshorne, Algebraic Geometry, Grad. Texts in Math. 52, Springer, New York, 1977.

[8] A. Schinzel, On reducible trinomials, Dissertationes Math. 329 (1993); Errata, Acta Arith. 73 (1995), 399-400.

[9] B. K. Spearman and K. S. Williams, On solvable quintics $x^{5}+a x+b$ and $x^{5}+a x^{2}+b$, Rocky Mountain J. Math. 26 (1996), 753-772.

[10] W. Trinks, Ein Beispiel eines Zahlkörpers mit der Galoisgruppe PSL(3,2) über $\mathbb{Q}$, manuscrit, Univ. Karlsruhe, 1968.

[11] H. Weber, Lehrbuch der Algebra, Vieweg, Braunschweig, 1895-1896.

Département de Mathématiques et Informatique

XLIM (UMR CNRS 6172)

Université de Limoges

123 avenue Albert Thomas

87060 Limoges Cedex, France

E-mail: julien.angeli@xlim.fr 